\title{
Electron-phonon coupling in iron-doped yttrium aluminum garnet
}

\author{
A. Anedda,${ }^{1,2}$ C. M. Carbonaro,,${ }^{1}$ D. Chiriu,${ }^{1}$ R. Corpino,${ }^{1}$ M. Marceddu,${ }^{1}$ and P. C. Ricci ${ }^{1, *}$ \\ ${ }^{1}$ Department of Physics, University of Cagliari, s.p. 8, km 0.700, 09042 Monserrato, Italy \\ ${ }^{2}$ LIMINA Laboratory, University of Cagliari, s.p. 8, km 0.700, 09042 Monserrato, Italy
}

(Received 20 September 2006; published 13 December 2006)

\begin{abstract}
The electron-phonon coupling in iron-doped yttrium aluminum garnet crystals is studied in the theoretical framework of linear and quadratic coupling. The line shift and line broadening of the zero phonon line of iron centers as a function of temperature between 10 and $150 \mathrm{~K}$ is qualitatively and quantitatively explained. The spectroscopic features of the line, deduced from the theory of lattice relaxation and multiphonon transitions, is compared with experimental results. The perturbation model indicates a linear coupling of medium strength and a strong quadratic coupling, providing an understanding of the thermal properties of spectral lines of ions in solids. The evaluated Huang-Rhys parameter is 2.05 and the quadratic coupling factor is $|w|=0.85$. The estimated Debye temperature is about $450 \mathrm{~K}$.
\end{abstract}

DOI: 10.1103/PhysRevB.74.245108

PACS number(s): 63.20.Kr, 65.40.-b, 78.60.-b

\section{INTRODUCTION}

Yttrium aluminum garnet (YAG) is one of the most important crystal hosts for solid-state lasers. When doped with transition-metal ions and rare-earth elements technological applications range from medical to industrial fields, from telecommunications to cutting edge academic and industrial research. ${ }^{1-4}$ Among the different possible doping ions, the sensitizing effect of $\mathrm{Fe}^{3+}$ is well known and was investigated in the past decades $;{ }^{5-11}$ iron ions contribute to the optical absorption of YAG crystal near the band edge, thus modifying the ultraviolet (UV) optical properties of the host material. As a result, for example, in neodymium and iron codoped YAG crystal, the dependence of the luminescent efficiency on the spectral distribution of the pumping flash lamps is reduced because of energy transfer between $\mathrm{Fe}^{3+}$ and $\mathrm{Nd}^{3+}$ ions. ${ }^{6,8,9}$

The YAG crystal $\left(\mathrm{Y}_{2} \mathrm{Al}_{5} \mathrm{O}_{12}\right)$ belongs to the space group $\operatorname{Ia} d=\mathrm{O}_{h}^{10}$, with $\mathrm{Al}^{3+}$ ions coordinated to nearest-neighboring oxygens with octahedral and tetrahedral point symmetry. The iron ions substitute for aluminum ions in the $a$ and $d$ sites with point symmetry $G_{a}=3 C_{i}$ and $G_{d}=S_{4}$, with octahedral and tetrahedral oxygen coordination, respectively. ${ }^{5,12}$ Since the radius of $\mathrm{Fe}^{3+}$ is larger than that of $\mathrm{Al}^{3+}$ ion, the local lattice structure surrounding the dopant ion $\left(\mathrm{Fe}^{3+}\right)$ is different from that for $\mathrm{Al}^{3+}$ ion it replaces, and an expansion distortion effect has been recently reported. ${ }^{5}$ Besides the modification induced in the absorption edge of the crystal host in the UV range, absorption bands in the visible (at 407 and $415 \mathrm{~nm}$ ) and emission in the near-infrared region (NIR) are related to $\mathrm{Fe}^{3+}$ centers. ${ }^{6,10,13} \mathrm{In}$ addition, $\mathrm{Fe}^{3+}$ easily forms covalent bonds with its ligands, giving rise to a charge-transfer transition between the molecular orbital level of the iron ion and the oxygen. Since the crystalline field at the iron ions depends on the local strain, the spectral features of the dopants can be regarded as a probe of the interaction between the iron ions and the local crystalline field. Static and dynamic strain causes spectral line broadening and line shift which are temperature dependent. Thus, in the case of the $\mathrm{Fe}^{3+}$ centers, the interaction between the dopant ions and the host crystal, which is expressed by the electron-phonon coupling (EPC), can be studied by investigating the spectral line shift and broadening of the NIR emission of iron ion centers. The topic is relevant not only from a fundamental point of view but also from a technological one, since the energy transfer between iron and neodymium ions in codoped YAG crystals is related to the EPC of $\mathrm{Fe}^{3+}$ centers. In addition, the subject is also important for the characterization of the yttrium iron garnet (YIG). When in the garnet structure the aluminum is replaced with iron, the YIG crystal is formed. ${ }^{14}$ YIG is a ferrimagnetic material that is widely used in various microwave and optical communication devices and other applications, mainly because of its suitable magnetic and magnetooptical properties. ${ }^{15-18}$ Indeed, YIG exhibits a large Faraday effect, high $Q$ factor at microwave frequencies, low absorption in the infrared wavelength region up to $600 \mathrm{~nm}$, and very small line width in electron spin resonance. ${ }^{16,19}$

In this paper we studied the EPC of $\mathrm{Fe}^{3+}$ ions in YAG crystals to understand the energy-level structure and the processes of energy transfer by optical centers formed by iron ions. In the past, different studies were reported on the coupling parameters of lanthanide ions in garnet crystals by means of linewidth broadening of the zero phonon line (ZPL) emission. ${ }^{20,21}$ These studies were carried out by investigating the thermal dependence of the shape and position of the ZPL, that is by analyzing the quadratic coupling between electrons and phonons.

In the present work, to have a complete understanding of the temperature dependence of the electron-phonon interactions, the linear and quadratic contribution to the EPC were both taken into account. The analysis of the linear and quadratic EPC constants of $\mathrm{Fe}^{3+}$ ions in YAG crystal was performed by studying the position, intensity, and width of ZPL and vibronics as a function of the temperature in the range $10-150 \mathrm{~K}$. It is shown that the study of the ZPL is a reliable and a relatively easy method to study the electron-phonon coupling strength.

\section{THEORETICAL BACKGROUND}

The EPC is the interaction between the electronic transitions of the emitting centers and the phonons of the host 
crystal. $^{22-26}$ At $T=0 \mathrm{~K}$ and $\vec{k}=0$, no phonons are involved in the transition; that is, the ZPL transition is purely electronic, and the $n=0$ vibrational state is the only occupied state. The intensity of the ZPL can be expressed as a Dirac delta function, ${ }^{24-26}$

$$
I_{k=0}\left(T_{0 \mathrm{~K}}, E\right) \propto \delta(\Delta E-E),
$$

where $T_{0 \mathrm{~K}}$ is the temperature at $0 \mathrm{~K}$ and $\Delta E$ is the energy difference between the fundamental level and the excited level.

In addition to the ZPL transition, there is also a finite probability that, in the final state, not only the vibronic modes of the impurity atom or molecule but also those of the host system become excited. Transitions that correspond to a change in phonon state of the lattice $(\vec{k} \neq 0)$ are called multiphonon transitions. Multiphonon transitions, in which $n$ phonons participate, will lead to the appearance of a broad phonon sideband accompanying the ZPL.

In order to understand the thermal dependence of the transition due to the interaction with phonons, it is necessary to consider both the contributions related to the linear and quadratic coupling between electron and phonons.

In the linear coupling approximation, and in the limit of low temperatures, the basic features of the ZPL and the phonon sideband can be interpreted by the value of the HuangRhys parameter $S,{ }^{24}$

$$
\frac{A_{\mathrm{ZPL}}}{A_{\mathrm{ZPL}}+A_{\mathrm{MP}}} \cong e^{-S}
$$

where $A_{\mathrm{ZPL}}$ and $A_{\mathrm{MP}}$ are the integrated area of the ZPL and the multiphonon transitions, respectively. The Huang-Rhys factor gives the size of the vibrational relaxation energy and the strength of the electron-phonon coupling. ${ }^{24-26}$

When the temperature increases, the Debye approximation at finite temperature can be applied, ${ }^{24,26}$

$$
\begin{aligned}
\frac{A_{\mathrm{ZPL}}}{A_{\mathrm{ZPL}}+A_{\mathrm{MP}}} & =\exp \left\{-S\left[1+4\left(\frac{T}{T_{D}}\right)^{2} \int_{0}^{T_{D} / T} \frac{x}{e^{x}-1} d x\right]\right\} \\
& \cong \exp \left\{-S\left[1+\left(\frac{2 \pi^{2}}{3}\right)\left(\frac{T}{T_{D}}\right)^{2}\right]\right\},
\end{aligned}
$$

where $T_{D}$ is defined as the Debye temperature and the approximation is valid in the limit of $T \ll T_{D}$.

In the low temperature limit, Eq. (3) reduces to Eq. (2). It follows from the above expressions that, when the temperature is increased, the integrated intensity of the ZPL has to decrease. By measuring the integrated intensity of the emission spectrum as a function of the temperature, one can determine the Huang-Rhys parameter $S$ and the Debye temperature $T_{D}$. However, in the linear approximation of EPC, the position and the line shape of the ZPL do not depend on temperature and the interaction between electrons and optical phonons does not produce a shift of the ZPL. ${ }^{25,27}$ Therefore, it is necessary also to consider the quadratic interaction terms between electrons and acoustic phonons to explain the broadening and the shift of the ZPL as the temperature increases.
The spectroscopic properties (line position and line shape) of ZPL depend on the temperature $(T)$ and are determined by several contributions. The peak position $E(T)$ can be expressed as $21,23,25,28$

$$
E(T)=E\left(T_{0 \mathrm{~K}}\right)+\Delta E^{D}+\Delta E^{R}+\Delta E^{\mathrm{Orb}},
$$

where the contributions to the line position due to onephonon or direct process $\left(\Delta E^{D}\right)$, two-phonon Raman process $\left(\Delta E^{R}\right)$, and two-phonon resonant Raman process or Orbach process $\left(\Delta E^{\mathrm{Orb}}\right)$ were considered. These contributions, and their relative weight to the temperature dependence of the ZPL peak position, are discussed in the following.

The term $E\left(T_{0 \mathrm{~K}}\right)$ is the position of the ZPL at $T=0 \mathrm{~K}$.

The contribution $\Delta E^{D}$ is related to the direct transition with the absorption or the emission of one phonon. It can be expressed as ${ }^{21}$

$$
\Delta E^{D}=\gamma_{1}\left(\frac{T}{T_{D}}\right)^{2} P \int_{0}^{T_{D} / T} \frac{x^{3}}{\left(e^{x}-1\right)\left\{x^{2}-\left[\left(E_{j}-E_{1}\right) / k_{B} T\right]^{2}\right\}} d x
$$

where $\gamma_{1}$ expresses the linear coupling parameter for the transition between the $E_{1}$ and $E_{j}$ states, $k_{B}$ is the Boltzmann's constant, $T_{D}$ is the Debye temperature, and $P$ indicates the principal value of the integral. The expression above is evaluated in the Debye approximation of the phonon density by assuming that the phonon density $\rho(\omega)$ scales with $\omega^{2}$, the square of the phonon frequency. ${ }^{26}$ The numerical value of the Debye temperature $T_{D}$ can be determined from the phonon cutoff energy, $\hbar \omega_{\text {cutoff }}$ (see Sec. IV). The contribution of the direct process to the line shift depends on the spectral separation of the two energy levels and the phonon cutoff energy of the crystal. A blueshift is reported when $\left(E_{j}-E_{1}\right)$ is comparable or larger than $\hbar \omega_{\text {cutoff }}$ and a redshift is observed in the other cases. As a general rule, the contribution to the thermal dependence of $\Delta E^{D}$ can be neglected with respect to the Raman one because the energy difference between $i$ and $j$ states is too large to be covered with a direct process. ${ }^{21,23,25,28}$

In the Raman two-phonon process, $\Delta E^{R}$, the system (at the stationary level $i$ ) absorbs a phonon that bring the system to level $j$ ' then the system relaxes to the stationary level $j$. The energy differences $\Delta E_{i j^{\prime}}$ and $\Delta E_{j^{\prime} j}$ are much larger than the phonon energies in the crystal. By using the Debye approximation of phonon energies, it can be derived that the temperature-dependent contribution to the line position due to the Raman process is described by ${ }^{21,24,25}$

$$
\Delta E^{R}=\alpha_{E}\left(\frac{T}{T_{D}}\right)^{4} \int_{0}^{T_{D} / T} \frac{x^{3}}{e^{x}-1} d x
$$

where

$$
\alpha_{E}=\frac{k_{B} T_{D}}{\hbar} \frac{3}{4 \pi} \frac{w}{w+1}
$$

is the electron-phonon coupling parameter for the Raman process and $w$ is the quadratic coupling constant $(w>-1){ }^{21,23-25,28}$ 
The contribution of the Orbach process $\left(\Delta E^{\mathrm{Orb}}\right)$ arises from a resonant Raman two-phonon process, when the energy differences between levels $i, j$, and $j^{\prime}$ are in the range of the phonon energy of the crystal.

In a similar way to the line shift, different mechanisms should be considered to explain the line broadening with temperature, but the minor contribution of different terms can be neglected. The line width of the zero phonon line is determined by the lifetime of the starting and final levels. Linear electron-phonon interactions alone do not produce line broadening, neither does the coupling to the optical phonons, nor the thermal expansion. ${ }^{29}$ In addition, the phonon-phonon interactions are, in general, weaker than the electron-phonon coupling for optical transitions.

The expression of the dependence of the linewidth from the temperature contains only contributions from the inhomogeneous line width $\left(\Gamma^{\text {inh }}\right)$, the one-phonon direct process $\left(\Gamma^{D}\right)$, the Raman two-phonon process $\left(\Gamma^{R}\right)$, and the radiative transition $\left(\Gamma^{\mathrm{rad}}\right),{ }^{21,23,25,28,30}$

$$
\Gamma(T)=\Gamma^{\mathrm{inh}}+\Gamma^{D}(T)+\Gamma^{R}(R)+\Gamma^{\mathrm{rad}}(T) .
$$

The inhomogeneous broadening $\Gamma^{\mathrm{inh}}$ is mainly due to strain and defects in the host lattice and, to first approximation it is possible to consider it as temperature independent. Due to the random probabilities of recombination due to the inhomogeneous processes, the curve shape is Gaussian.

$\Gamma^{D}$ is the contribution due to the direct or one-phonon process: one phonon is emitted or absorbed. This contribution is described by ${ }^{21,31}$

$$
\Gamma^{D}(T)=\Gamma_{e m}^{D}(T)+\Gamma_{a s}^{D}(T)=\sum_{j<i} \beta_{i j}(n+1)+\sum_{j>i} \beta_{i j}(n) .
$$

In this equation, $\beta_{i j}$ is a coefficient of the EPC for the direct process, $n=\left[\exp \left(\Delta E_{i j} / k_{B} T\right)-1\right]^{-1}$ is the phonon occupation number, $i$ and $j$ are two vibrational energy levels. The energy gap between the states $i$ and $j$ is in the range of the phonon energies of the system. Direct processes cause Lorentzian broadening of the line shape because of the energydependent one-phonon relaxation rate.

By using the Debye approximation for the phonon energies, the contribution of Raman two-phonon relaxation can be expressed by

$$
\Gamma^{R}(T)=\alpha_{\Gamma}\left(\frac{T}{T_{D}}\right)^{7} \int_{0}^{T_{D} / T} \frac{x^{6} e^{x}}{\left(e^{x}-1\right)^{2}} d x
$$

where

$$
\alpha_{\Gamma}=\frac{k_{B} T_{D}}{\hbar} \frac{9}{4}\left(\frac{w}{w+1}\right)^{2},
$$

is the EPC parameter for the Raman process. ${ }^{21,23-25,28,30}$

The last term, $\Gamma^{\mathrm{rad}}$, is the contribution due to the radiative relaxation of a state.

\section{SAMPLES AND EXPERIMENTAL SETUP}

Photoluminescence (PL) and excitation of PL (PLE) measurements were carried out at the SUPERLUMI experimen-

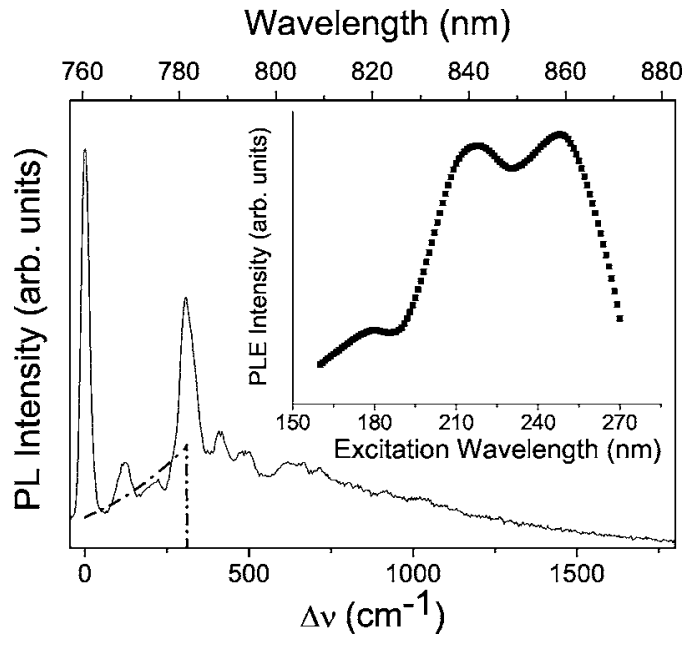

FIG. 1. PL spectrum of ZPL and vibronics at $10 \mathrm{~K}$. The inset reports the PLE spectrum of the ZPL at $10 \mathrm{~K}$. The dash-dotted line shows the fit for the phonon spectrum by using the Debye model.

tal station on the I beamline of the HASYLAB synchrotron laboratories at DESY (Hamburg) by using the pulsed synchrotron radiation (SR) for excitation. The PLE measurements were performed in the $4-10 \mathrm{eV}$ energy range with $0.3 \mathrm{~nm}$ of bandwidth. Excitation spectra were corrected for the spectral efficiency of the excitation source by using a sodium salicylate standard. The PL signal was dispersed by a $0.5 \mathrm{~m}$ Czerny-Turner monochromator and detected in the $1.5-5.0 \mathrm{eV}$ energy range with a charge-coupled device (CCD). Emission spectral bandwidth was $0.1 \mathrm{~nm}$. PL and PLE spectra were recorded under multibunch operation and detected with an integral time window of $192 \mathrm{~ns}$ correlated to the SR pulses. A continuous-flow liquid-helium cryostat was used to vary the temperature of the sample chamber in the $8-150 \mathrm{~K}$ range.

Yttrium aluminum garnet samples were grown by Scientific Materials Europe (Italy) by the Czochralski method. Pure oxides of $\mathrm{Al}_{2} \mathrm{O}_{3}(99.999 \%)$ and $\mathrm{Y}_{2} \mathrm{O}_{3}(99.999 \%)$ were mixed and presintered under pressure of $140 \mathrm{MPa}$ and processed at $1400{ }^{\circ} \mathrm{C}$ for $24 \mathrm{~h}$. Sintered tablets were melt at $1970{ }^{\circ} \mathrm{C}$ in an iridium crucible and pulled at $0.8 \mathrm{~mm} / \mathrm{h}$ with angular velocity $\omega_{r}=15(\delta y / \delta x) \mathrm{rpm}$ under Ar atmosphere. Samples were cut along the [111] direction and lapped to an optical finish.

\section{RESULTS AND DISCUSSION}

In Fig. 1, the emission spectrum at $10 \mathrm{~K}$ of the $\mathrm{Fe}^{3+}$ ions in YAG crystal is reported. The excitation wavelength was $\lambda=180 \mathrm{~nm}$, corresponding to the fundamental absorption of the host material. ${ }^{10}$ The spectrum shows the ZPL centered at $763.0 \mathrm{~nm}$ and the phonon repetitions of the ZPL (vibronics) between 770 and $790 \mathrm{~nm}$. The emission properties of $\mathrm{Fe}^{3+}$ ions in $\mathrm{Y}_{3} \mathrm{Al}_{5} \mathrm{O}_{12}$ are well known and were previously analyzed by Voitukevich et al. ${ }^{10}$ These bands derive from the transitions between the fundamental term ${ }^{6} A_{1}(S)$ and the term ${ }^{4} T_{1}(G)$ of the $\mathrm{Fe}^{3+}$ ions. The observation of four finestructure components of the ${ }^{4} T_{1}(G)$ emission term in the lu- 


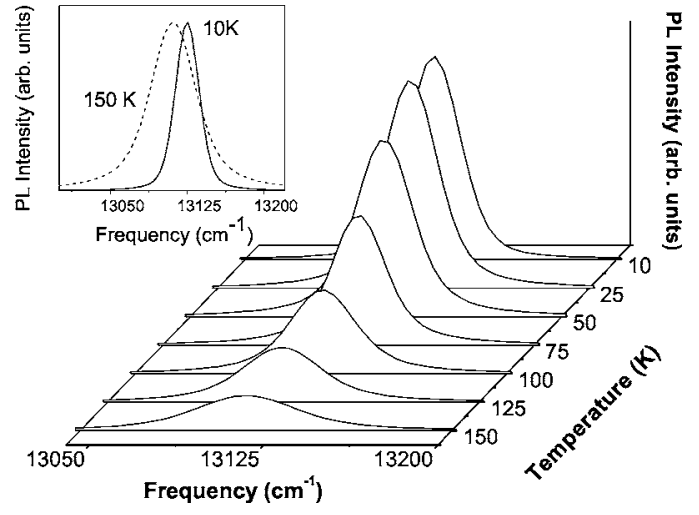

FIG. 2. PL spectra of the ZPL as a function of the temperature in the $10-150 \mathrm{~K}$ range. The inset shows the normalized PL spectra at 10 and $150 \mathrm{~K}$.

minescence spectrum allowed us to determine the nature of the crystal field experienced by the $\mathrm{Fe}^{3+}$ ions in the matrix by discriminating the coordination symmetry of the lattice position. The emission features were ascribed to transitions from tetrahedral $\mathrm{Fe}^{3+}$ sites. ${ }^{8,10}$

The inset of the figure presents the PLE spectrum of the ZPL in the range between 160 and $270 \mathrm{~nm}$. The spectrum shows an intense band in the $230-270 \mathrm{~nm}$ region, in good agreements with previous results. ${ }^{13}$ This excitation band corresponds to the principal absorption of the $\mathrm{Fe}^{3+}$ ions that constitutes the preferential charge-transfer channel in the wavelength range around $800 \mathrm{~nm}$. The excitation band at $260 \mathrm{~nm}$ is indicated as the convolution of two excitation bands relative to $\mathrm{Fe}^{3+}$ ions in tetrahedral and octahedral sites. The peak position of the ZPL is independent excitation wavelength in the investigated range.

The PL spectra were recorded at different temperatures over the range between $10 \mathrm{~K}$ and $150 \mathrm{~K}$ (Fig. 2). As the temperature increases, the pattern of the luminescence spectrum changes sharply and, as previously reported by Voitukevich et al. ${ }^{10}$ it is possible to observe a redshift of the ZPL peak, a substantial broadening of the PL bands and a decrease of the relative intensity of the ZPL band. The inset of Fig. 2 shows the normalized PL spectra of the ZPL at 10 and $150 \mathrm{~K}$ to better evidence the line shift and broadening. Above $150 \mathrm{~K}$ the emission bands overlap and the analysis by means of deconvolution is not possible. Thermal quenching of the ZPL can be hypothesized to explain the absence of ZPL signal above $150 \mathrm{~K}$. At room temperature the spectrum presents a structureless intense broad band peaked at about $810 \mathrm{~nm}$ whose shape is asymmetric in the long-wavelength side, with a total width of about $1600 \mathrm{~cm}^{-1}$ (here not shown). In addition, the presence of a band centered at $760.6 \mathrm{~nm}$ whose relative intensity with respect to the intensity of the ZPL increases as the temperature increases was also reported. ${ }^{10}$ Since the ${ }^{4} T_{1}(G)$ levels are very close the one to each other, the integrated area ratio between the ZPL and the band at $760.6 \mathrm{~nm}$ is well described by Boltzmann's law.

Figure 3 shows the dependence of the ratio between the integrated area of the ZPL and the whole emission spectrum as a function of temperature. The theoretical curve according to Eq. (3) is also reported. The best fit result (linear correlation factor $R>0.95$ ) gives, for the two adjustable parameters $T_{D}$ and $S$, the values of $468 \mathrm{~K}$ and 2.05 , respectively. This value of the Huang-Rhys factor represents an EPC of medium strength $(1<S<6)$, where the recombination processes are dominated by transitions to vibrational levels with $\vec{k}>0$. The ZPL is, however, still observable. The Debye temperature $T_{D}$ also can be estimated by means of the Debye model, $T_{D}$ being a measure for the effective maximum phonon energy. In the Debye model, the phonon density increases proportional to $\omega^{2}$ and then drops to zero at the phonon cutoff frequency $\omega_{\text {cutoff. The }}$ determination of this parameter can be performed by a graphical method, ${ }^{18}$ as illustrated in Fig. 1. This method consists in the determination of the cutoff frequency, $\omega_{\text {cutoff }}$, in the PL spectrum, as corresponding to the maximum of the intensity of the vibronics. At this frequency, the phonon densityprofile drops to zero. The Debye temperature is given by the expression

$$
T_{D}=\frac{\hbar \omega_{\text {cutoff }}}{k_{B}}
$$

As illustrated in Fig. 1, the phonon density increases to a maximum up to $310 \mathrm{~cm}^{-1}$, in agreement with the Debye model. By assuming this value as $\omega_{\text {cutoff }}$, the temperature $T_{D}$ is estimated as $410 \mathrm{~K}$ in reasonable agreement with the value previously reported. The difference between the two values of the Debye temperature is due to the fact that the real density of the phonon states does not fit exactly the Debye model.

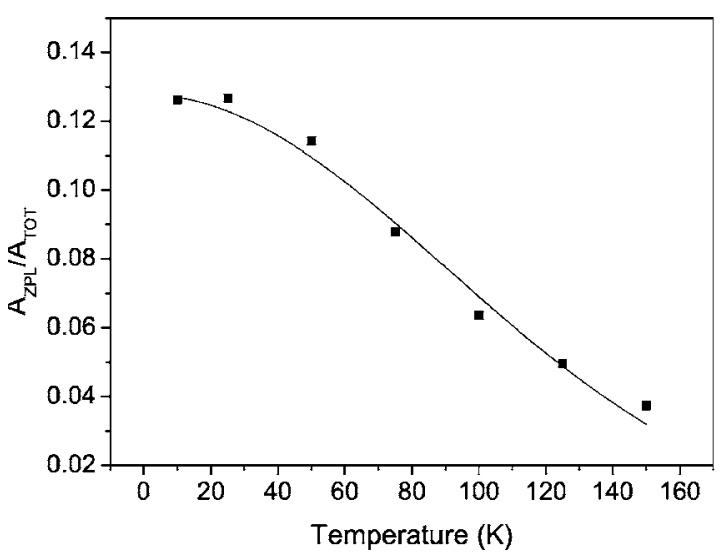

FIG. 3. Dependence of the ratio between the integrated area of the ZPL and the whole emission spectrum as a function of temperature. The solid line reproduces the best fit result according to Eq. (3). 


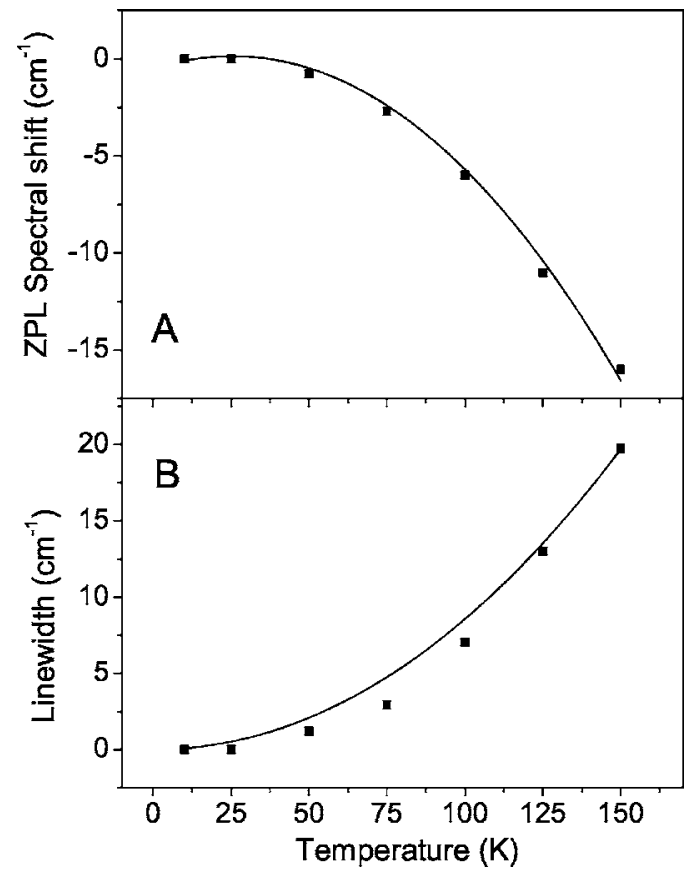

FIG. 4. Line shift (a) and line broadening (b) of the ZPL as a function of the temperature. The solid lines reproduce the best fit results according to Eqs. (13) and (14), respectively.

In Figs. 4(a) and 4(b), the spectral line shift and the broadening of the total line width [full width at half maximum (FWHM)] of the ZPL in $\mathrm{Y}_{3} \mathrm{Al}_{5} \mathrm{O}_{12}: \mathrm{Fe}^{3+}$ are reported as a function of the temperature.

In most studies the contribution of the Orbach process as well as of other minor contributions to line shift (such as multiphonon relaxation) are neglected. ${ }^{21,28,30}$ In addition, if the peak position of the ZPL is independent of excitation energy, the contribution to the shift from localized modes may be neglected. ${ }^{24}$ It turns out that the main contributions to the shift of the ZPL originate from quadratic interactions between electrons and acoustic phonons. Hence, one can use the theoretical expression obtained in the Debye model to discuss electron-phonon interactions. ${ }^{24,25}$

$E(T)=E\left(T_{0 \mathrm{~K}}\right)+\Delta E^{R}=E(T=0)+\alpha_{E}\left(\frac{T}{T_{D}}\right)^{4} \int_{0}^{T_{D} / T} \frac{x^{3}}{e^{x}-1} d x$.

It has been shown that an increase in temperature causes both blue- and redshifts, depending on the material. ${ }^{24,25}$ The direction and magnitude of the temperature shift of the ZPL depends on the relation between the shift due to thermal expansion of the crystal lattice and the shift due to electronphonon interactions at constant volume. The shift of the ZPL due to thermal expansion of the lattice occurs to the blue, while the shift caused by electron-phonon interactions occurs to the red with increasing temperature. ${ }^{24,25}$

As the temperature increases, the ZPL shows a redshift of about $16 \mathrm{~cm}^{-1}$ between 10 and $150 \mathrm{~K}$ [Fig. 4(a)], indicating that the thermal expansion can be reasonably neglected. In other words, when the temperature is well below the Debye temperature, the sample can be regarded as a very "hard" crystal. In this case, the shift due to EPC considerably exceeds the shift caused by the thermal expansion of a crystal, determining the whole experimentally observed line shift.

Figure 4(a) reports the shift of the ZPL as a function of the temperature and the theoretical curve calculated according to Eq. (13). The fit was performed by using the quadratic coupling constant, $w$, and the Debye temperature as free parameters (linear correlation factor $R>0.99$ ). The results for the two variables are $450 \mathrm{~K}$ and -0.85 respectively. The Debye temperature obtained is in good agreement with the values obtained from the linear coupling analysis and from the Debye approximation. As expected, $w$ is larger than $-1 .^{24}$ Since a weak quadratic coupling is expressed by a value of $w$ close to zero, the calculated one $(|w|=0.85)$ indicates a relative high quadratic coupling as expected by the relative large shift of the ZPL with temperature when compared to the variation of the ZPL of the $\mathrm{Cr}^{3+}$ in the YAG structure in the same temperature range. ${ }^{20}$ The study of the ZPL of $\mathrm{Cr}$ in YAG crystals reported for the $\alpha_{E}$ parameter a value of $330 \mathrm{~cm}^{-1}$ and a Debye temperature of $550 \mathrm{~K}$. The corresponding $w$ value is -0.6 .

Figure 4(b) reports the line broadening of the ZPL in the temperature range between 10 and $150 \mathrm{~K}$. The FWHM was estimated by means of a deconvolution process with Voigt profiles $^{23,24}$ and the line-width data were fitted according to the theory reported in Sec. II. Actually, Voigt profiles of the emission bands were chosen because of the mixed contribution of homogeneous and inhomogeneous broadening of the spectral lines. It is well known that a physical process that has the same probability of occurrence for all the atoms of the system produces a Lorentzian line shape (homogeneous broadening), while a physical process that has a random distribution of occurrence for each atom produces a Gaussian line shape (inhomogeneous broadening). If both type of broadening processes are present, the line shape is the convolution of Lorentzian and Gaussian contributions represented by a Voigt profile..$^{23,24}$

In general, the contribution of direct processes to line broadening is neglected since the evaluation of the electronphonon strength is not influenced by the incorporation of the direct process in the computation. ${ }^{23}$ In addition, in the present case of Fe:YAG, the presence of a large number of crystal field components makes the calculation of the $\beta_{i j}$ parameter hard to perform correctly. Moreover, since the radiative relaxation time is of the order of some microseconds, ${ }^{9}$ the radiative relaxation term in Eq. (8) can be neglected. According to the Heisenberg principle, microseconds lifetimes broaden the total width less than $10^{-6} \mathrm{~cm}^{-1}$. Therefore, the temperature dependence of the line width of the ZPL can be expressed as

$$
\Gamma(T)=\Gamma^{\mathrm{inh}}+\Gamma^{R}(T)=\Gamma^{\mathrm{inh}}+\alpha_{\Gamma}\left(\frac{T}{T_{D}}\right)^{7} \int_{0}^{T_{D} / T} \frac{x^{6} e^{x}}{\left(e^{x}-1\right)^{2}} d x .
$$

Equation (14) was applied to fit the line width of the ZPL as a function of the temperature by assuming the coupling parameters and the Debye temperatures as adjustable param- 
eters. The values obtained for $\alpha_{\Gamma}$ and for the Debye temperature were $328.9 \mathrm{~cm}^{-1}$ and $450 \mathrm{~K}$, respectively. Although the theoretical curve reproduces the trend of the experimental points at different temperatures and the fitting parameters are in agreement with the previous results of YAG crystals $\mathrm{Cr}^{3+}$ doped, ${ }^{20}$ there is not a perfect match between the experimental data and the proposed model. This fact is clearly showed by deducing the value of the coupling constant $w$ from Eq. (11), $|w|=0.46$, as compared to the value of 0.85 calculated in the line-shift fitting procedure. The disagreement is due to the different approximation used in this analysis, such as the assumption that the inhomogeneous broadening is temperature independent, the neglecting of the direct recombination, and the calculation of only the Raman two-phonon process as possible dephasing mechanism. Moreover, the EPC is not the same for all phonon modes and can be in principle different for phonons contributing to line-broadening processes compared to those contributing to line-shifting processes. Except for this discrepancy, the perturbation approach described here has been very successful in providing an understanding of the thermal properties of spectral lines of ions in solids. ${ }^{30}$

A final comment pertains to the fact that the above expressions for the spectral line shift and line broadening, Eqs. (13) and (14), respectively, are calculated by assuming the Debye model. One should bear in mind that the actual phonon distribution is far from the model, but the thermal dependence is often well described by a single characteristic temperature that can be interpreted as an effective Debye temperature that may not coincide with the value extracted from heat measurements because not the whole set of phonons is coupled with the ions.
The performed study shows a relatively easy method to study the EPC strength in $\mathrm{Fe}^{3+}$ doped YAG crystals and the model can be extended to other materials. Although a more rigorous nonperturbative method can be developed to explain the temperature dependences of spectral line widths and position, the simpler mathematics of the perturbation approach described here, provides a better insight into the physical processes that are taking place.

\section{CONCLUSIONS}

In this work, we studied the electron-phonon coupling in iron-doped yttrium aluminum garnet crystals. By analyzing the experimental data in the theoretical framework of linear and quadratic coupling, the line shift and line broadening of the zero phonon line of iron centers as a function of the temperature in the $10-150 \mathrm{~K}$ is qualitatively and quantitatively explained. The applied model, based on a perturbative approach, indicates a linear coupling of medium strength and a strong quadratic coupling, providing an understanding of the thermal properties of spectral lines of ions in solids. The evaluated Huang-Rhys parameter is 2.05 and the quadratic coupling factor is $|w|=0.85$. The estimated Debye temperature is about $450 \mathrm{~K}$.

\section{ACKNOWLEDGMENTS}

This work was supported by the European CommunityResearch Infrastructure Action under the FP6 "Structuring the European Research Area" Programme (through the Integrated Infrastructure Initiative "Integrating Activity on Synchrotron and Free-Electron-Laser Science").
*Author to whom correspondence should be addressed. Email address: carlo.ricci@dsf.unica.it

${ }^{1}$ M. Grinberg, A. Sikorska, A. Sliwiski, J. Barzowska, Y. R. Shen, S. B. Ubizskii, and S. S. Melnyk, Phys. Rev. B 67, 045113 (2003).

${ }^{2}$ Y. N. Xu, Y. Chen, S. D. Mo, and W. Y. Ching, Phys. Rev. B 65 , 235105 (2002).

${ }^{3}$ A. G. Okhrimchuk and A. V. Shestakov, Phys. Rev. B 61, 988 (2000).

${ }^{4}$ M. Nomura Shin-ichiro, T. Harada, and K. Yoshikawa, Phys. Rev. Lett. 88, 093903 (2002).

${ }^{5}$ Die Dong, Kuang Xiao-Yu, Guo Jian-Jun, Wang Hui, and Zhou Kang-Wei, Phys. Rev. B 72, 073101 (2005).

${ }^{6}$ S. R. Rotman, C. Warde, H. L. Tuller, and J. Haggerty, J. Appl. Phys. 66, 3207 (1989).

${ }^{7}$ Y. F. Chen, K. T. Wu, Y. D. Yao, C. H. Peng, K. L. You, and W. S. Tse, Microelectron. Eng. 81, 329 (2005).

${ }^{8}$ M. V. Korzhik, M. G. Livshits, B. I. Minkov, and V. B. Pavlenko, Sov. J. Quantum Electron. 22, 24 (1992).

${ }^{9}$ M. V. Korzhik, M. G. Livshits, M. L. Meilman, B. I. Minkov, and V. B. Pavlenko, Opt. Spectrosc. 70, 471 (1991).

${ }^{10}$ Yu. A. Voitukevich, M. V. Korzhik, V. V. Kuzmin, M. G. Livshits, and M. L. Meilman, Opt. Spectrosc. 63, 810 (1987).
${ }^{11}$ M. V. Korzhik, M. G. Livshits, Kh. S. Bagdasarov, A. M. Kevorkov, T. A. Melkonyan, and M. L. Meilman, Sov. J. Quantum Electron. 19, 344 (1989).

${ }^{12}$ J. P. Hurrell, S. P. S. Porto, I. F. Chang, S. S. Mitra, and R. P. Bauman, Phys. Rev. 173, 851 (1968).

${ }^{13}$ M. L. Meil'man, M. V. Korzhik, V. V. Kuzmin, M. G. Livshits, Kh. S. Bagdasarov, and A. M. Kevorkov, Sov. Phys. Dokl. 29 (1), 61 (1984).

${ }^{14}$ G. B. Scott, D. E. Lacklison, and J. L. Page, Phys. Rev. B 10, 971 (1974).

${ }^{15}$ R. Urban, A. Putilin, P. E. Wigen, S.-H. Liou, M. C. Cross, P. C. Hammel, and M. L. Roukes, Phys. Rev. B 73, 212410 (2006).

${ }^{16}$ A. S. Tatarenko, G. Srinivasan, and M. I. Bichurin, Appl. Phys. Lett. 88, 183507 (2006).

${ }^{17}$ Y. V. Khivintsev, Bijoy K. Kuanr, Ian Harward, R. E. Camley, and Z. Celinski, J. Appl. Phys. 99, 08P512 (2006).

${ }^{18}$ G. A. Melkov, V. I. Vasyuchka, Yu. V. Kobljanskyj, and A. N. Slavin, Phys. Rev. B 70, 224407 (2004).

${ }^{19}$ A. A. Semenov, S. F. Karmanenko, B. A. Kalinikos, G. Srinivasan, A. N. Slavin, and J. V. Mantese, Electron. Lett. 42, 641 (2006).

${ }^{20}$ A. P. Vink and A. Meijerink, J. Phys. Chem. Solids 61, 1717 (2000). 
${ }^{21}$ A. Ellens, H. Andres, A. Meijerink, and G. Blasse, Phys. Rev. B 55, 173 (1997).

${ }^{22}$ Baldassarre Di Bartolo, Optical Interactions in Solids (Wiley, New York, 1968), Chap. 15.

${ }^{23}$ Richard C. Powell, Physics of Solid-State Laser Materials (Springer-Verlag, New York, 1998), Chaps. 4-5.

${ }^{24}$ H. Wang, F. D. Medina, D. D. Liu, and Y. D. Zhous, J. Phys.: Condens. Matter 6, 5373 (1994).

${ }^{25}$ J. L. Skinner and D. Hsu, Adv. Chem. Phys. 65, 1 (1986).
${ }^{26}$ G. P. Srivastava, The Physics of Phonons (Adam Hilger, IOP, New York, 1990), Chaps. 2-7.

${ }^{27}$ D. Hsu and J. L. Skinner, J. Chem. Phys. 81, 1604 (1984).

${ }^{28}$ M. G. Beghi, C. E. Bottani, and V. Russo, J. Appl. Phys. 87, 1769 (2000).

${ }^{29}$ T. E. Jenkins, J. Phys. Chem. 19, 1065 (1986).

${ }^{30}$ D. E. McCumber and M. D. Sturge, J. Appl. Phys. 34, 1682 (1963).

${ }^{31}$ J. L. Skinner, J. Chem. Phys. 77, 3398 (1981). 